\title{
Development of a Methodology to Assess Mechanical Impulse Effects Resulting from Lightning Attachment to Lightweight Aircraft Structures
}

\author{
C. A. Featherston ${ }^{1, a}$, M. Eaton ${ }^{1, b}$, S. L. Evans ${ }^{1, c}$, K. M. Holford ${ }^{1, d}$, R. Pullin ${ }^{1, e}$ \\ and M. Cole $\mathrm{C}^{2, \mathrm{f}}$ \\ ${ }^{1}$ Cardiff School of Engineering, Cardiff University, Queens Buildings, The Parade, Cardiff, \\ CF24 3AA, UK \\ ${ }^{2}$ EADS Innovation Works, The Quadrant, Celtic Springs, Coedkernew,Newport, NP10 8FZ, UK \\ ${ }^{a}$ FeatherstonCA@cardiff.ac.uk, ${ }^{b}$ EatonM@cardiff.ac.uk, ${ }^{\mathrm{c}}$ EvansSL6@cardiff.ac.uk, \\ dHolford@cardiff.ac.uk, ${ }^{\mathrm{e}}$ PullinR@cardiff.ac.uk, ${ }^{\mathrm{f}}$ Matthew.Cole@eads.com
}

Keywords: Lightning, lightweight structures, digital image correlation, finite element analysis.

\begin{abstract}
The effect of lightning attachment to structures and vehicles is a cause of major concern to a number of different industries, in particular the aerospace industry, where the consequences of such an event can be catastrophic. In 1963, a Boeing 707 was brought down in Maryland killing 81 people on board, triggering the improvement of lightning protection standards. However, commercial jets are still struck on average once every 10,000 hours of flight time and between 1963 and 1989 forty lightning related accidents were recorded within the U.S.A alone. The rapid increase in the use of composite materials in aircraft design and the consequent increase in complexity when determining the effects of a lightning strike, has led to new challenges in aircraft protection and the requirement for improved understanding and standardisation.
\end{abstract}

The attachment of lightning to a structure causes damage through three mechanisms. Primarily a supersonic acoustic shock wave, caused by rapid heating of the arc channel during initial attachment, resulting in a large and rapid overpressure. Secondly a magnetic force generated by the fields developed in the high current areas around the lightning attachment point. Finally a mechanism specifically related to composite materials, where the rapid vaporisation of an expanded copper foil layer (designed to quickly transmit current across a structure, thus reducing its focus) trapped between the composite material and the protective paint layers causes an additional overpressure, which is exacerbated by additional paint layers acting to contain the explosion and direct it inwards.

The work described in this paper looks to develop a technique to measure these forces in order to better understand and assess their effects. A novel methodology has been developed to allow the estimation of peak overpressure forces produced by the acoustic shock wave resulting from the attachment. The methodology utilises Digital Image Correlation and ultra-high speed photography to acquire full-field displacement measurements of panel deflections at frame rates of up to $1,000,000$ frames per second. The experimental results are used in the optimisation of a finite element model, outputting the parameters of an acoustic shock that produces representative displacements, velocities and accelerations. The method is currently being validated by performing a series of tests on aluminium panels subject to instrumented impact testing. The next stage will be to use the technique developed to aid a program of investigation into the effects of artificial lightning strike events on aluminium and composite panels.

\section{Introduction}

Lightning parameters A lightning strike is essentially a high amplitude direct-current pulse. Its waveform is well-defined and is divided into four parts known as components A to D as shown in Fig. 1. Component $\mathrm{A}$ is a high-current pulse. It is a direct current transient typically in the order of $200 \mathrm{kA}$ lasting for $50 \mu \mathrm{s}$ and having a current rise rate of $3 \times 10^{10} \mathrm{~A} / \mathrm{s}$. Component $\mathrm{B}$ is a transition 
phase generally in the order of $2 \mathrm{kA}$. Component $\mathrm{C}$ is a continuing current of approximately 200$800 \mathrm{~A}$ and lasting up to $0.75 \mathrm{~s}$. The last component, $\mathrm{D}$, is a restrike surge which is typically half the amplitude of component $\mathrm{A}$ in a given strike and has the same duration as component $\mathrm{A}$. On average 3 or 4 restrikes will occur in one lightning event but where the maximum observed is 26 restrikes in one event.

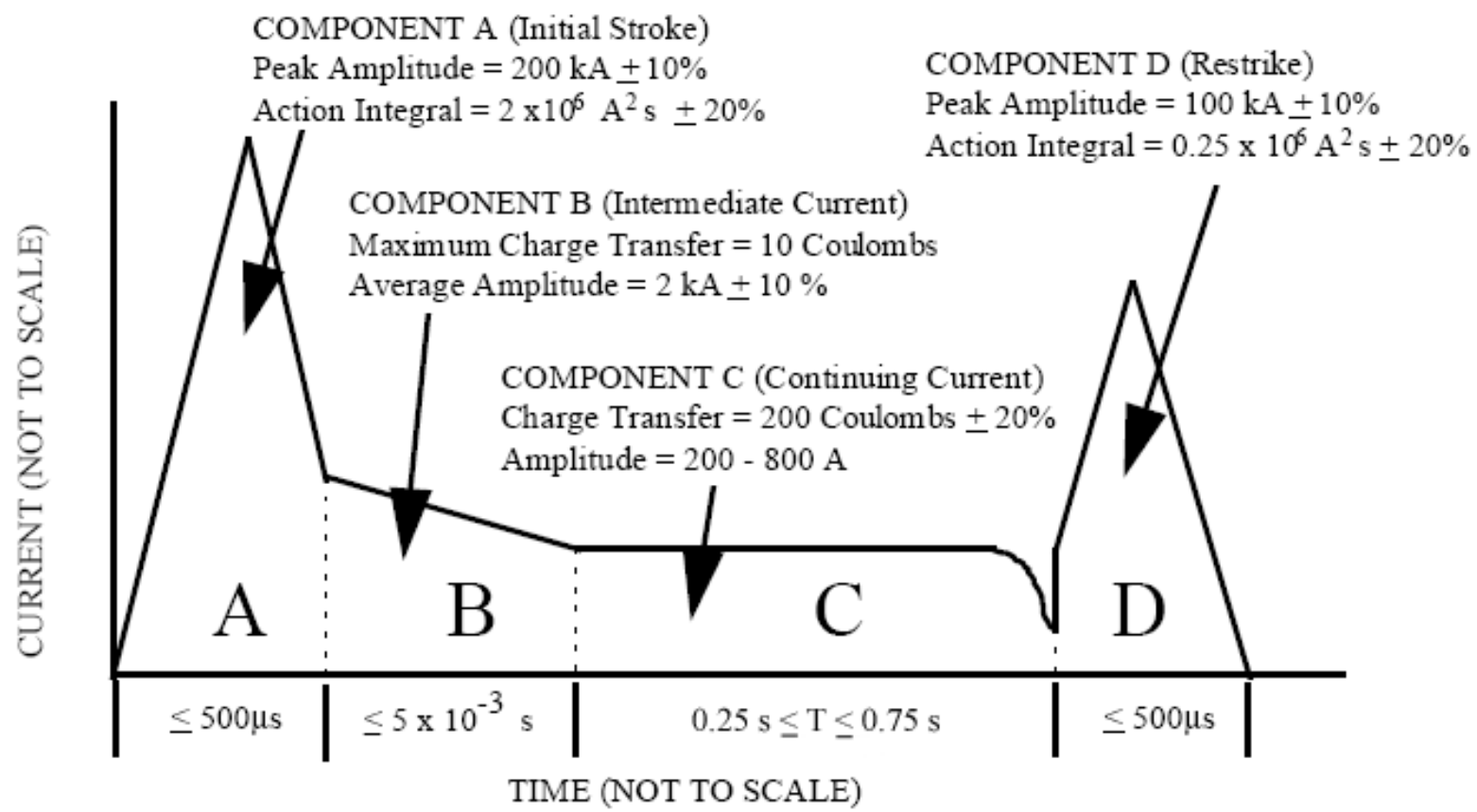

Figure 1 Lightning waveform [1]

Indirect effects The multiphysics nature of the lightning strikes causes a range of different effects including heating, a pressure wave resulting from the acoustic shock and magnetic forces.

On initial attachment the high current return stroke generates a large acoustic shock wave caused by the rapid heating of the lightning arc which propagates cylindrically at an initially supersonic speed. The resulting axial pressure at the root of the arc can cause damage in the form of permanent deformation or in the case of composites matrix cracking. Initial estimates of this overpressure and its propagation for a 30kA stroke have been made by Hill [2] who calculated a maximum overpressure of $30 \mathrm{MPa}$ at a radial distance of $10 \mathrm{~mm}$ from the point of attachment, reducing to 20 $\mathrm{MPa}$ at $20 \mathrm{~mm}$ and $10 \mathrm{MPa}$ at $40 \mathrm{~mm}$. However in order to fully understand the effects of this pressure wave for different levels of lightning strike, this data must be derived for a range different currents.

Further forces are generated due to the magnetic fields developed around the point of attachment of the arc. Since following attachment current flows away from this point in many directions each of which can be considered as a separate current path and when two or more electric currents flow in the same direction through two or more parallel wires (or paths), attractive forces act on the wires (or the different parts of the structure), forces are generated which can deflect the structure. Although it is generally believed that this effect is much smaller than the effect of the acoustic shock wave it must still be quantified in order to understand the total effects of the lightning strike.

Finally the localised heating occurring in the vicinity of the attachment point can also cause problems particularly in the presence of paint finishes and in composites the Expanded Copper Foil layer due to the generation of explosive forces as explained previously. This also needs to be quantified. 
Only by gaining an insight into the effects of these distinct forces and their interdependencies can the effects of different levels of lightning strike be adequately predicted. The work presented in this paper represents a pilot study to determine the feasibility of using an explicit finite element model describing the response of a specimen during lightning attachment in order to determine the mechanical effects of such a strike. This model, once optimised using experimental data on full field deformation and hence velocity and acceleration profiles during impact obtained using high speed DIC will provide the loading parameters required.

\section{Methodology}

Due to the level of complexity involved in such a multi-phenomenal event in which the energy input due to the various effects has the ability to cause a combination of elastic and potentially plastic deformation and damage and which is truly dynamic and therefore incorporates inertial effects it was concluded that direct measurement of the forces involved was not feasible. For this reason a methodology was developed in which indirect effects such as full-field displacement and hence the velocity and acceleration of the plate would be measured using DIC. Until recently measurements of this kind would not have been possible due to limitations in available camera hardware, i.e. maximum frame rates achievable at a suitable resolution. However with the advent of Ultra-High speed cameras, such as the Shimadzu HPV-2, suitable images can now be captured at up to $1 \mathrm{M}$ fps on a single CCD chip. High speed DIC has previously been utilised to monitor dynamic fracture events in polymers [4] and to aid the calculation of material parameters at high strain rates using the virtual fields method [5]. The full-field data would then be used to optimize the parameters within a dynamic FEA model created using a combination of the pre and post processor Oasys Primer and D3 Plot and the analysis code LS-DYNA. The technique would be validated based on a simple test involving impacting a metallic plate. This methodology is illustrated in Fig. 2.

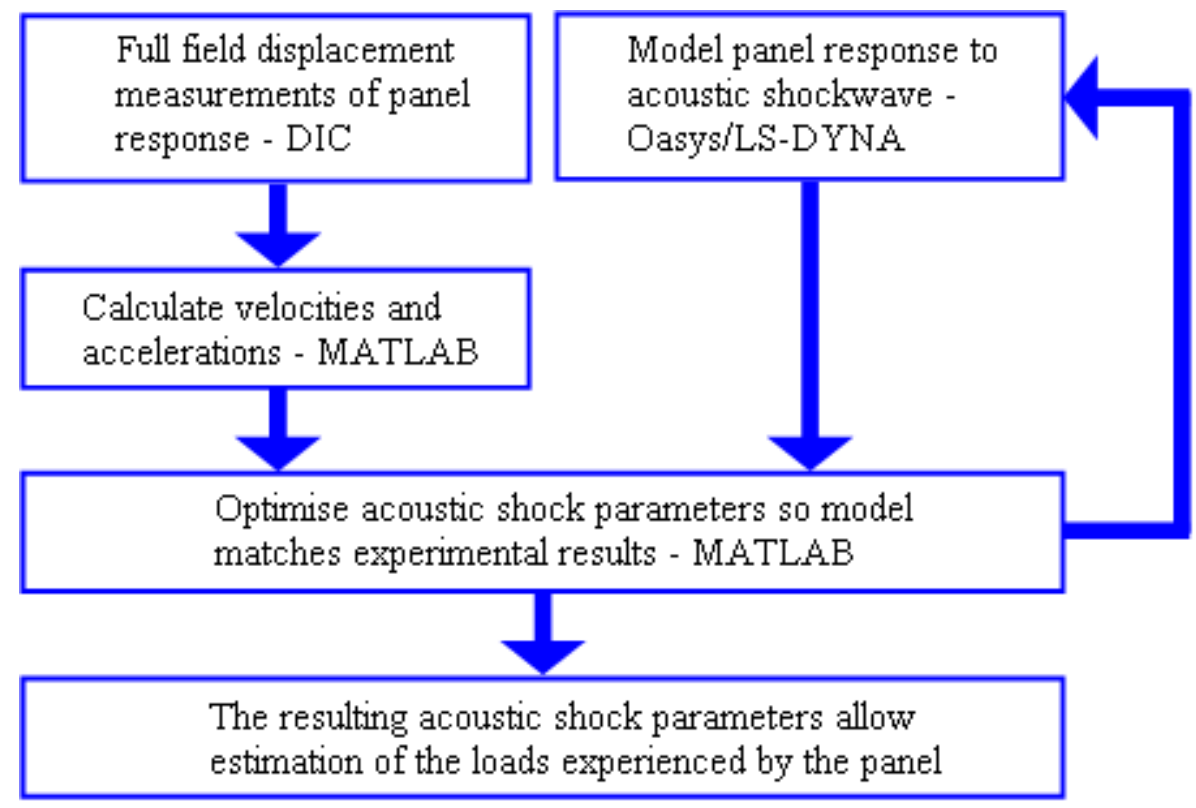

Figure 2 Methodology

\section{Experimental Work}

Set-up As stated, the methodology is being validated based on a high speed impact test on a rectangular plate. The plate was manufactured from $0.6 \mathrm{~mm}$ thick aircraft grade duraluminium BS1470 6082-T6 with an unsupported length of $287 \mathrm{~mm}$ and a width of $254 \mathrm{~mm}$ (based on the dimensions of a typical aerospace panel (Jegley [3])). It was built-in along all four edges by bolting 
through the plate to a pair of frames $25 \mathrm{~mm}$ x $25 \mathrm{~mm}$ in cross section. Full field displacement data was captured using digital image correlation. This was performed using VIC 3D (Correlated Solutions Inc, USA) system, capturing data at a rate of 1000fps using a pair of AOS Technologies MOTIONeer high speed cameras synchronised using Correlated Solution's VIC Snap software to gather data for post processing using VIC 3D software. The test was performed using a Instron Dynatup $9250 \mathrm{HV}$ accelerated drop test machine, with a series of impacts based on dropping a mass of $5.7 \mathrm{~kg}$ from a variety of heights from 0.05 to $0.2 \mathrm{~m}$. The test set-up is illustrated in Fig. 3 .

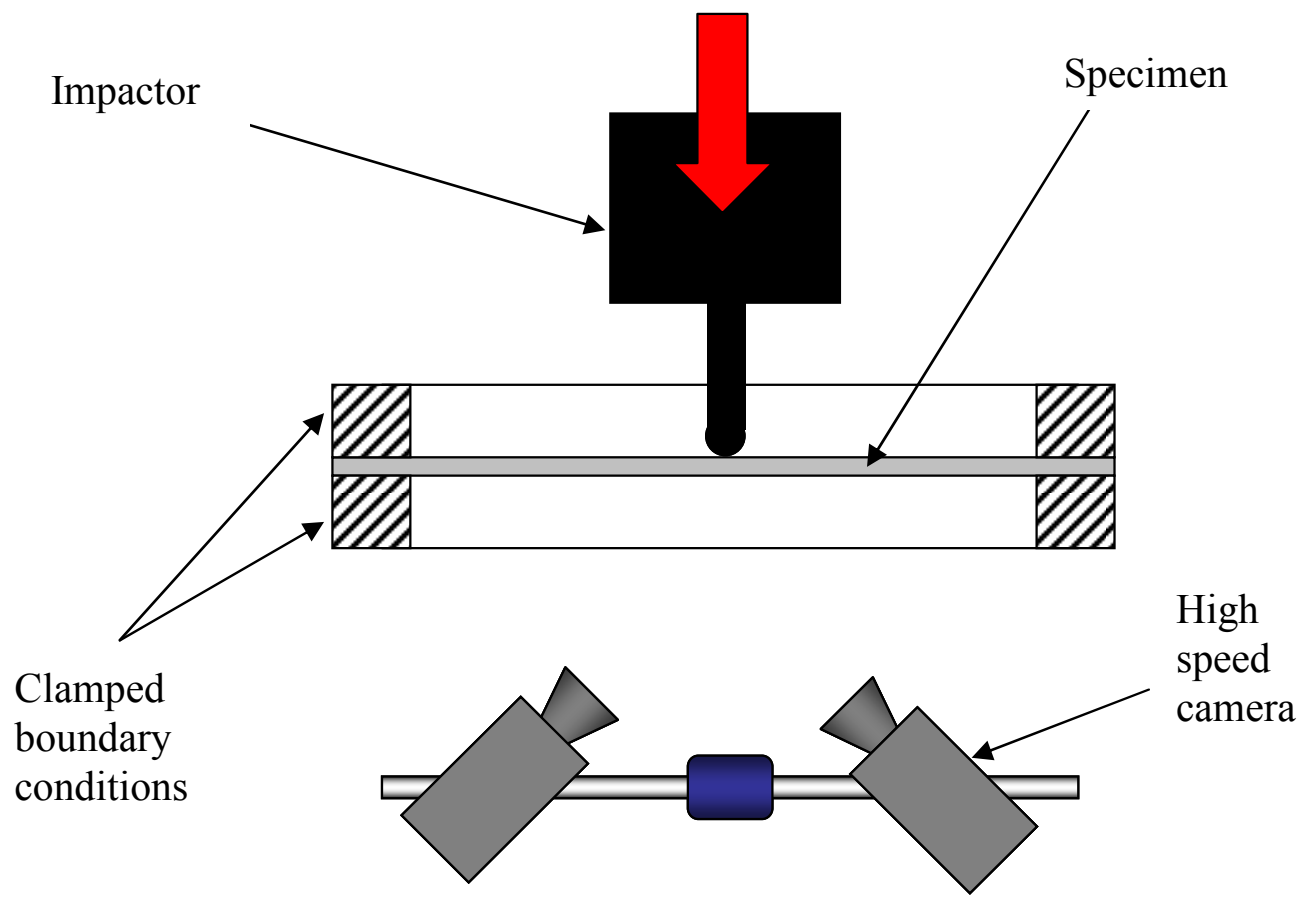

Figure 3 Schematic of drop test set-up

Test results A series of profiles of displacement against time at the point of impact taken from the results of the digital image correlation are presented in Fig. 4, for drop heights of $0.05,0.1$ and $0.2 \mathrm{~m}$. Velocity profiles again corresponding to behaviour at the point of impact are presented in Fig.5. Finally full field displacement contours taken from the tests performed with a drop height of $0.2 \mathrm{~m}$ corresponding to different times during the impact event are provided in Fig. 6.

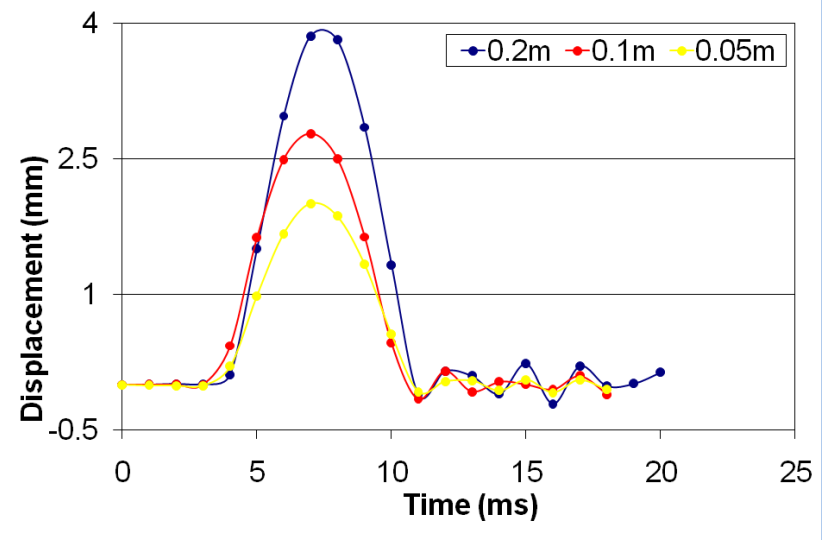

Figure 4 Displacement at the point of impact

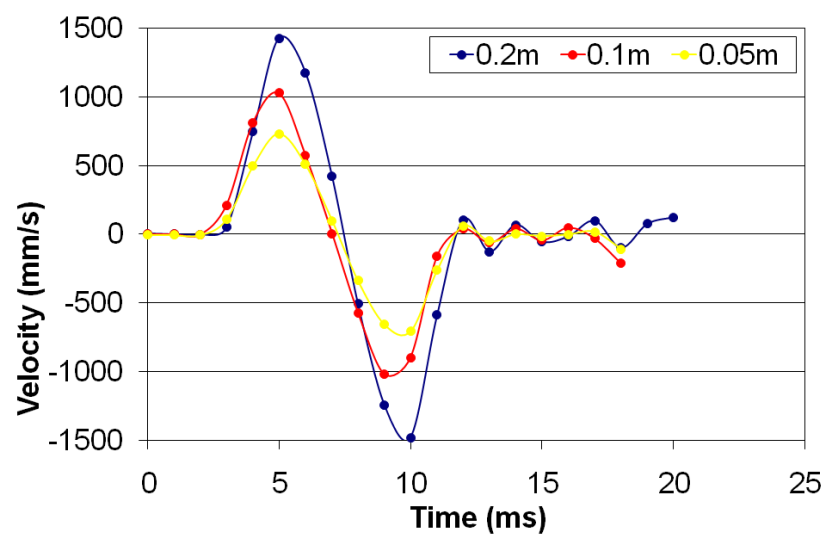

Figure 5 Velocity at the point of contact 

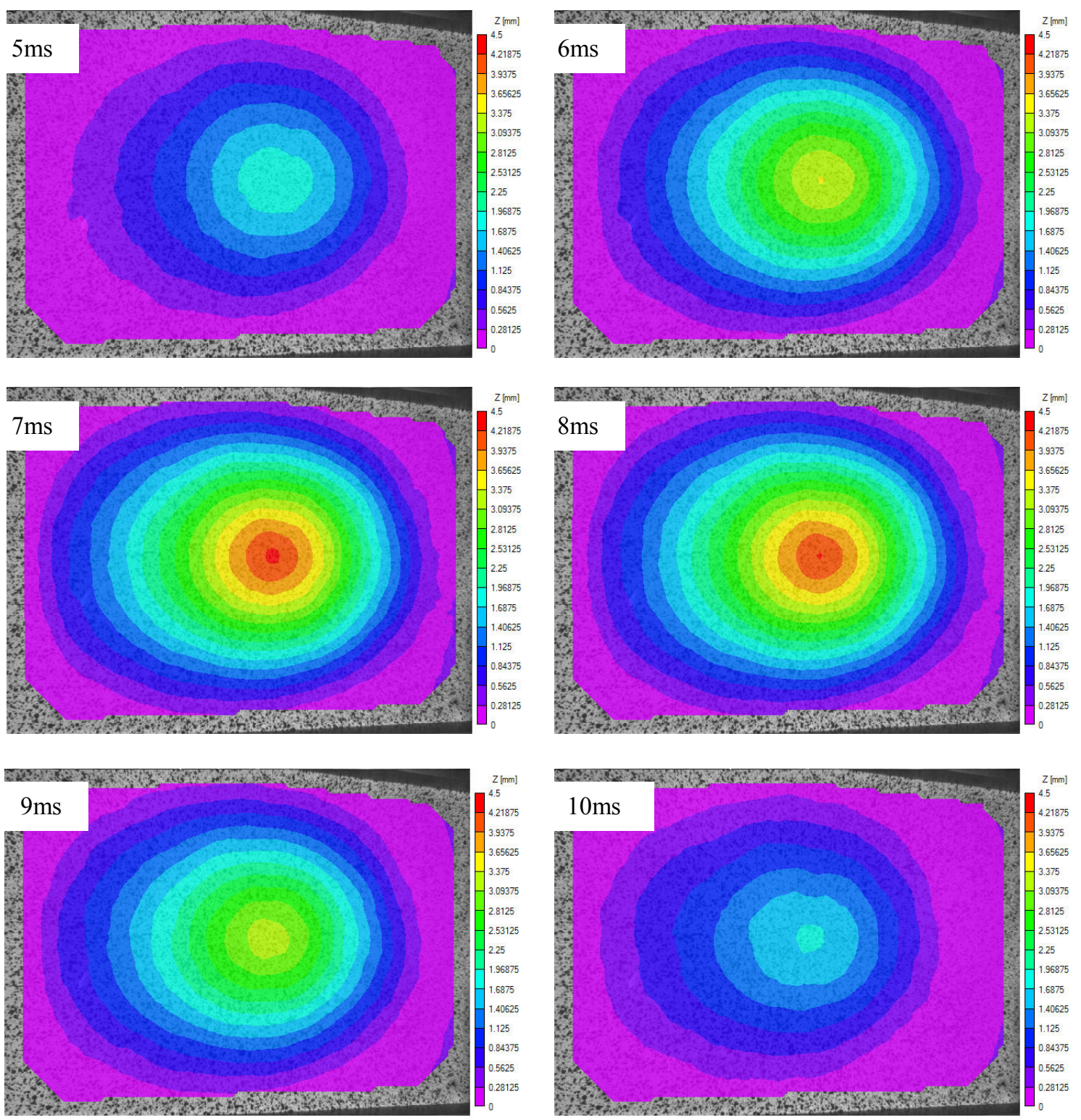

Figure 6 Full field displacement contours

\section{Modelling}

LS-Dyna is an explicit solver developed for the analysis of high speed short duration events in which the effects of inertia are important. It is capable of analysing time steps of less than $1 \mu$ s. Initially developed to aid crash testing in the automotive industry Dyna is capable of dealing with linear and non-linear transient dynamics, non-linear material behavior, contact surfaces and crucially for this work solid-fluid interactions. Utilising these features LS-Dyna has been used to model blast effects and explosive forming of sheet metals and hence is ideally suited to the proposed application.

As discussed above initial validation of the proposed methodology for measuring the effects of lightning strike is being conducted using an aircraft grade aluminium panel subject to a drop weight impact. Utilising symmetry, impact at the centre of the panel has been represented based on a one quarter model. To represent the impactor, a rigid body with the geometry of a sphere the same diameter as the impact tup and the equivalent mass of the impact carriage is placed a small distance above the panel and given an initial velocity which can be varied to represent different drop heights. An image of the model is presented in Fig. 7. 


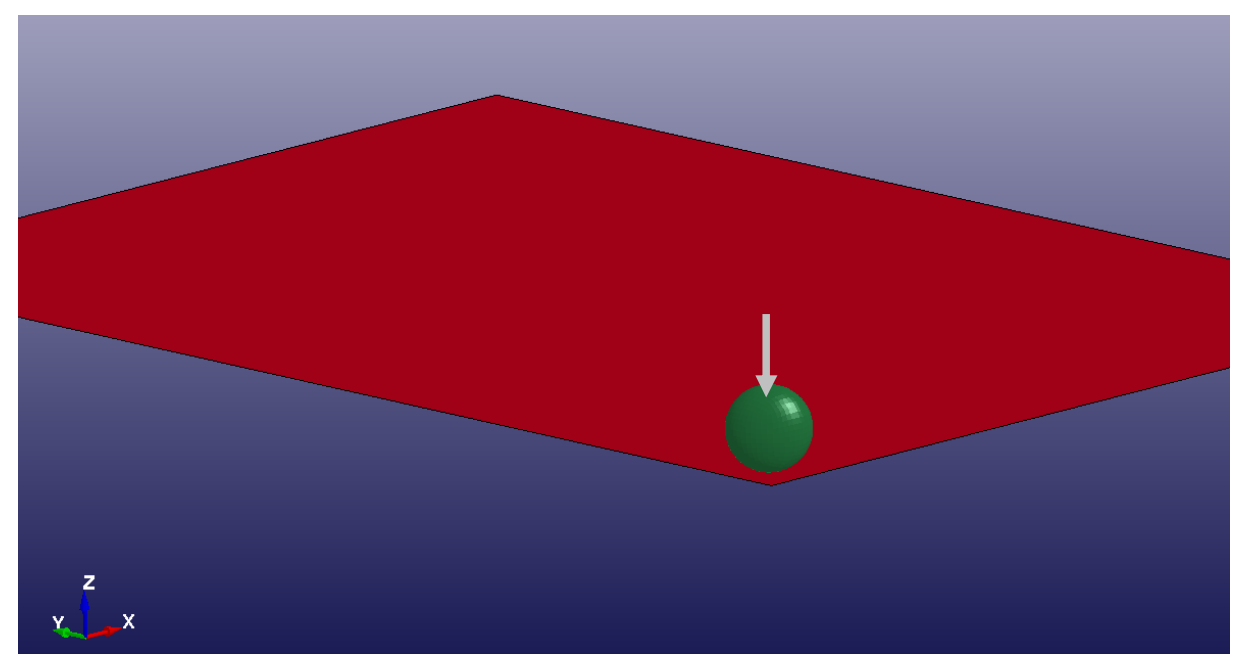

Figure 7 Finite Element model of panel impact.

\section{Optimisation}

The aim of the optimisation process in this context is to minimise the difference between the experimental measurements and the FEA results by adjusting parameters related to loading. This is achieved through use of a minimisation function in MatLab which measures the difference between the experimental and FEA data sets, be those displacements, velocities or accelerations, and minimises this difference by iteratively adjusting the loading parameters. The resulting parameters provide an estimation of the load experienced by the panel. In this case this is the initial velocity of the impact sphere, however once validated the same approach can be extrapolated to determine for example parameters describing the acoustic shock.

\section{Conclusions}

A methodology has been developed to enable the forces experienced by a structure during lightning strike to be determined using indirect effects which can be measured. A pilot study is being performed to validate this methodology based on a metallic plate subject to impact from a drop test rig. A series of tests based on different impact energies have been performed. Full field displacement and velocity data have been successfully obtained using high speed digital image correlation. A model of the experimental set-up has been developed using the explicit finite element analysis code LS-Dyna. Loading parameters within the model are currently being optimized to obtain a fit with the experimental data. Comparison of the loading parameters with measured data will validate the suitability of the technique for the measurement of load.

\section{References}

[1] Department of Defence: Electromagnetic Environmental Effects, Military Handbook 464, (1997).

[2] R.D. Hill: Channel Heating in Return Stroke Lightning, Geophys., Vol. 76, (1971).

[3] D.C. Jegely: Behavior of compression-loaded composite panels with stringer terminations and impact damage. AIAA/ASME/ASCE/AHS 39th Structures, Structural Dynamics, and Materials Conference, Long Beach, California, (1998).

[4] M. S. Kirugulige, H. V. Tippur and T. S. Denney: Measurement of transient deformations using digital image correlation method and high-speed photography: application to dynamic fracture. Appl. Opt., Vol. 46 (22), (2007).

[5] S. Avril, F. Pierron, M. A. Sutton and J. Yan : Identification of elasto-visco-plastic parameters and characterisation of Lüders behaviour using digital image correlation and the virtual fields method. Mechanics of Materials, Vol. 40, (2008). 